\title{
A STUDY OF THE ELECTRON IMAGE DUE TO IONIZING EVENTS IN A TWO-DIMENSIONAL LIQUID ARGON TPC WITH A 24 CM DRIFT GAP
}

S. Bonetti ${ }^{3}$, A. Braggiotti ${ }^{4}$, E. Buckley ${ }^{2 \dagger}$, M. Campanella ${ }^{3}$, G. Carugno', G. Cecchet ${ }^{5}$, P. Cennini ${ }^{1}$, S. Centro ${ }^{4}$, A. Ciocio ${ }^{2}$, S. Cittolin ${ }^{1}$, B. Dainese ${ }^{4}$, M. Ferro-Luzzi ${ }^{1}$, F. Gasparini ${ }^{4}$, A. Gonidec ${ }^{1}$, P.F. Manfredi ${ }^{3}$, E. Meroni ${ }^{3}$, R. Muñoz 1 , J-M. Perreau ${ }^{1}$, F.Pietropaolo ${ }^{4}$, F. Ptohos ${ }^{1}$, F. Ragusa ${ }^{3}$, P. Rossi ${ }^{4}$, C. Rubbia ${ }^{1}$, D. Schinzel ${ }^{1}$, W.F. Schmidt ${ }^{1 \ddagger}$, W. Seidl ${ }^{1}$

1 CERN, CH-1211, Geneva 23, Switzerland.

2 Laboratori Nazionali di Frascati, Via Enrico Fermi 40, Frascati, Rome, Italy.

3 Dipartimento di Fisica and Sezione dell'INFN, Univ. di Milano, Via Celoria 16, Milano, Italy.

4 Dipartimento di Fisica and Sezione dell'INFN, Univ. di Padova, Via F. Marzolo 8, Padova, Italy.

5 Dipartimento di Fisica and Sezione dell'INFN, Univ. di Pavia, Via A. Bassi 6, Pavia Italy.

$\dagger \quad$ Visiting Scholar, High Energy Physics Laboratory, Harvard University, Cambridge, MA 02138, USA.

₹ Visiting scientist 1986/1987 from the Hahn-Meitner Institut, Berlin, Fed. Rep. Germany.

* Present address, High Energy Physics Laboratory, Harvard University, Cambridge, MA 02138, USA.

Submitted to Nuclear Instruments and Methods in Physics Research A 


\begin{abstract}
We have tested a liquid argon Time Projection Chamber with a novel wire configuration based on electrostatic focussing which allows the realisation of a nondestructive detection of the electron image produced by ionizing events. The chamber was tested in a $5 \mathrm{GeV}$ pion beam at the CERN Proton Synchrotron. The measured pulse shapes at both $200 \mathrm{~V} / \mathrm{cm}$ and $500 \mathrm{~V} / \mathrm{cm}$ were in very good agreement with the expected shapes, calculated taking into account the electron lifetime, the response of the electronics and the longitudinal diffusion of the electron cloud. The measured electron drift velocity was in good agreement with the results of other workers as well as with our previous measurements.

We have also analysed a sample of events containing delta-rays in order to study the behaviour of low energy electrons in the liquid argon. We find that for electron energies greater that $5 \mathrm{MeV}$ the measured energy spectrum agrees very well with the predicted spectrum after corrections for acceptance and energy loss, hence demonstrating the feasibility of recognising low energy electrons in liquid argon.
\end{abstract}




\section{Introduction}

Since the initial proposal for a liquid argon Time Projection Chamber in 1977[1], much work has been done by different groups towards realising this kind of device[27]. Liquid argon is ideal for the detection of rare events because of its high density and its ability to be both target and detector. In particular, the proposed ICARUS detector[8] is a large volume liquid argon TPC which will be situated in the Gran Sasso Underground Laboratory in Italy and will study primarily solar neutrino interactions.

In a detector such as this it is desireable to reduce the number of detection planes to a minimum. This implies very long drift lengths, of the order of 2 metres, meaning that the liquid argon has to be free of electronegative impurities at a level of $<1 \mathrm{ppb}$. We have previously demonstrated that it is possible to obtain ultra-pure liquid argon with an electron lifetime in the region of $9 \mathrm{~ms}$ ( $0.03 \mathrm{ppb}$ oxygen equivalent)[9] using a simple purification system consisting of a mixture of molecular sieve and silica gel together with an Oxisorb cartridge[10] to remove oxygen. The design for the purification system was chosen as a result of the experience gained in cleaning warm liquids at CERN in connection with the new UA1 calorimeter[11].

As part of the continuing development program for the ICARUS experiment we have successfully tested a two-dimensional liquid argon TPC with a $24 \mathrm{~cm}$ drift gap. The chamber consisted of a single detection plane in the form of a grid of sense-wires. The wires plus the drift time provided a two-dimensional electron image of tracks produced in the drift volume. With this chamber we have demonstrated that the theory of charge induction on a grid of wires in a liquid noble gas works in practice, hence the idea of a non-destructive read-out has been realised for the first time. We note here that the group of H.H. Chen have also reported results from a two-dimensional TPC but their readout plane consisted of strips $2.5 \mathrm{~mm}$ apart etched on copper -plated G-30, rather than a grid of wires[4].

The chamber was tested in a $5 \mathrm{GeV}$ pion beam at the CERN Proton Synchrotron. This provided a much higher event rate than cosmic rays and allowed us to determine accurately the energy and direction of the incoming particle. To test the feasibility of identifying low energy electrons, we have studied the production of delta-rays in the chamber and measured their energy spectrum.

The paper is organised in the following way. In Section 2 we explain the improvements we have made to the original design for a liquid argon TPC $[1,6]$. We then describe the chamber constructed using this idea. In Section 3 we give a brief summary of the purification system as this has been explained in detail in a previous publication[9]. Section 4 deals with the electronics, the data accquisition and the experimental set-up. In Section 5 we discuss the chamber performance and results and in Section 6 we present our conclusions. 


\section{The chamber design}

The theory of electron imaging of ionizing events occuring inside the sensitive volume of a liquid argon TPC and the design of such a chamber was developed by Gatti and co-workers[6]. The basic design consisted of a screen-grid of equally spaced parallel wires and a sense-wire grid, also of equally spaced parallel wires of the same pitch, placed at a distance $\mathrm{W}$ from the screen-grid. This arrangement produces a twodimensional electron image, one co-ordinate is provided by the position of the hit wires and the other by the drift time. For a three-dimensional image, a second sense-wire grid is placed at $90^{\circ}$ to the first. The presence of the screen-grid means that a signal is not induced on the sense-wires until the drifting electrons cross the grid. The grid will be $100 \%$ transparent to the drifting electrons if the following condition is satisfied[12]

$$
\frac{E_{A}}{E_{B}}>\frac{(1+x)}{(1-x)}
$$

where $E_{A}$ is the field after the grid, $E_{B}$ is the field before the grid and

$$
x=\frac{2 \pi r}{d}
$$

where $\mathrm{r}$ is the wire radius and $\mathrm{d}$ is the distance between the wire centres. The principles of operation of this system have been extensively discribed in ref. 6 and will not be discussed further.

The original design suffered from two main problems. The first was that nonnegligible signals were induced on one sense-wire from electrons drifting towards the neighbouring sense-wire. One had to move at least three sense-wire gaps away before this signal became negligible. The second problem was non-uniformity in shape and magnitude of the induced signals, which depended strongly on the position of the drifting electrons with respect to the sense-wires.

In designing the chamber, we have modified the original design to obtain a wire arrangement that significantly reduces both of the above problems. This is shown schematically in Fig. 1. We have replaced the single sense-wire plane with a plane containing pairs of sense-wires separated by a distance $s$ with an extra wire in between each pair which acts as a shield between the sense-wires. We retain the screen-grid as before. The electric fields in the drift space, screen-grid/sense-wire gap and sensewire/anode gap are represented by $E_{1}, E_{2}, E_{3}$ respectively. For electric field $E_{2}$ larger than $E_{1}$ the field lines are focussed towards the sense-wires. If the electric field $E_{3}$ is chosen to be the correct value then the field lines pass between the sense-wires and end on the anode. For a three-dimensional chamber the field lines can pass to a second sense-wire plane hence realising a non-destructive readout. 
We have investigated different choices of the wire diameters and spacings and our final choice is given below

$$
\begin{gathered}
\mathrm{W}=2 \mathrm{~mm} \\
\mathrm{~d}=2 \mathrm{~mm} \\
\mathrm{~s}=0.5 \mathrm{~mm} \\
\mathrm{R}_{\mathrm{GW}}=50 \mu \mathrm{m}, \mathrm{R}_{\mathrm{SW}}=25 \mu \mathrm{m}
\end{gathered}
$$

where $R_{G W}$ and $R_{S W}$ are the radii of the grid wires and the sense-wires respectively. For these parameters the transparency of the screen-grid is $100 \%$ if the ratio of the fields after and before the grid, $E_{2} / E_{1}$ is at least 1.4. However, in order to focus the field lines so that the sense-wires are $100 \%$ transparent requires that $E_{2} / E_{1} \geq 3$. We have considered two possible configurations for the electric fields that satisfy this criteria, namely $E_{1}: E_{2}: E_{3} \equiv 1: 3: 9$ and $E_{1}: E_{2}: E_{3} \equiv 1: 5: 5$. We have developed a drift chamber simulation program that allows us to calculate the potential map, the electron trajectories and the signal shapes for a particular wire configuration. The calculations made with this program suggest that the second field configuration has better focussing properties and a simpler potential distribution with the screen-grid sitting at the natural potential given by the field while the sense-wires and shielding wires are at ground. The corresponding field map is shown in Fig. 2 for the parameters given above. The shielding inefficiency of the screen-grid, which is a function of the geometry only and not the electric field, is $\approx 10 \%$ [12]. This inefficiency causes the sense-wires to see the drifting electrons before they cross the screen-grid and results in a small tail at the beginning of the pulse.

As before, the signal induced on a single sense-wire varies according to the lateral position of the drifting electrons. However, for each pair of wires the sum of the two signals is almost constant as shown in Fig. 3a, where the sum is plotted as a function of the position of the drifting electrons with respect to the grid-wires. We note that the induced signal never reaches $100 \%$, the maximum value is $\approx 88 \%$ and the mean value is $\approx 75 \%$. This situation could be improved by using larger diameter wires or increased spacing between the screen-grid and the sense-wire grid. In Fig. $3 b$ we show the summed signal as a function of drift time for three positions of the drifting electrons, labelled A, B and C (as indicated in Fig. 3a). Even though the pairs of sense-wires are shielded from each other by the grid-wires the shielding is not $100 \%$. This is illustrated by the curves labelled D, E and F in Fig. 3b which are the signals induced on the sense-wire due to electrons drifting in the neighbouring cell. This contribution is $\leq 6 \%$ and that from the next but one cell is essentially zero, an improvement over the original design. 
A cross-section through the chamber is shown in Fig. 4. All of the metal parts were constructed from stainless steel and were subjected to the CERN 'standard cleaning procedure' which has been described in previous publications $[9,13]$. The chamber was constructed from a stainless steel cathode plate of $100 \times 100 \mathrm{~mm}^{2}$ which was joined to the wire plane by a series of 24 field-shaping rings also $100 \times 100 \mathrm{~mm}^{2}$ of $3 \mathrm{~mm}$ thickness separated by $7 \mathrm{~mm}$ spacers of insulating material (PTFE). Each ring was connected to the next by a $60 \mathrm{M} \Omega$ ceramic resistor. The resistors had their coat of varnish removed and their leads were gold-plated to prevent corrosion during cleaning. The resistors were cleaned with soap and water plus ultrasound. The final resistor was connected from the screen-grid to ground and it was possible to achieve the field ratio of $1: 5$ before and after the grid by using another $60 \mathrm{M} \Omega$ resistor. This racetrack ensured that the electric field inside the chamber was uniform. The grid- and sensewires were stainless steel of $100 \mu \mathrm{m}$ diameter and $50 \mu \mathrm{m}$ diameter respectively and were mounted in a MACOR[14] frame. There were 31 pairs of sense-wires. The distance between the screen-grid/sense-wire plane and the sense-wire plane/anode was $2 \mathrm{~mm}$.

The high voltage was supplied through the bottom of the chamber via a ceramic feed-through as can be seen in Fig. 4. The chamber was contained in a stainless steel vessel $388 \mathrm{~mm}$ long with DN160 ConFlat[15] flanges on the top and bottom and the total volume of the chamber was 8.6 litres. The top flange contained a single pipe for evacuation and filling of the chamber and four multi-pin cryogenic feed-throughs for the sense-wire readout. Each feed-through read out eight pairs of sense-wires. There were three additional feed-throughs, one for the anode high voltage and signal, one for the screen-grid high voltage and one for the sense-grid high voltage. These feedthroughs could also be used to supply a test-pulse to the anode and the grids. In the field configuration used it was not necessary to apply any external voltage to the grids.

The two flanges were sealed with copper O-rings and the whole assembly was baked-out under vacuum for 48 hours at $100^{\circ} \mathrm{C}$. A relatively low temperature was used to avoid damage to the ceramic resistors. After this procedure a typical leak test rate was $5 \times 10^{-9}$ mbar litre $\mathrm{s}^{-1}$ and the outgassing rate of the chamber was $1 \times 10^{-13} \mathrm{mbar}$ litre $\mathrm{s}^{-1} \mathrm{~cm}^{-2}$. Published values for the outgassing of stainless steel (obtained using different cleaning procedures) range from about $5 \times 10^{-12} \mathrm{mbar}$ litre $\mathrm{s}^{-1} \mathrm{~cm}^{-2}$ to $10^{-13}$ mbar litre $\mathrm{s}^{-1} \mathrm{~cm}^{-2}[16]$.

\section{The Purification System}

The purification system has been described in detail in a previous publication[9]. The same procedure for liquefaction was followed as described in ref.9, with the rate of liquefaction into the chamber being about 1 litre of liquid per hour. Prior to allowing 
any liquid into the chamber, the latter was slowly cooled until the external temperature of the pot reached about $-100^{\circ} \mathrm{C}$. This was a precaution to prevent wires breaking on coming into contact with the cold argon entering the chamber.

\section{Experimental Set-up: electronics and data-acquisition}

Data were taken with the chamber placed in a $5 \mathrm{GeV}$ pion beam at the CERN Proton Synchrotron (PS). A schematic of the experimental set-up and readout system is shown in Fig. 5. The trigger was defined by five scintillation counters placed in coincidence. The vertical spread of the beam entering the chamber was limited by a 'finger' counter of dimensions $80 \times 5 \times 5 \mathrm{~mm}^{3}$. The accelerator produced one burst of particles of $500 \mathrm{~ms}$ duration every 10 seconds and the number of triggers per burst in the five counters was $\approx 6$. This rate gave typically one track recorded in the chamber per trigger. The chamber was placed in an open dewar of liquid argon for ease of refilling and both were then placed on a platform which enabled the position and height of the chamber with respect to the beam to be adjusted.

A schematic of the front-end electronics together with the connections to the chamber is shown in Fig. 6. The signals induced on the sense-wires were brought out of the chamber through $120 \mathrm{~cm}$ long commercial coaxial cables. These long cables had the disadvantage of increasing the input capacitance of the amplifier to $120 \mathrm{pF}(110 \mathrm{pF}$ due to the coaxial cable and $10 \mathrm{pF}$ real detector capacitance) and also to make the amplifier more sensitive to microphonic noise. This type of noise had two main sources. The first was mechanical vibrations from outside the detector, this contribution was found to be negligible in the operational environment at the CERN PS test beam. The second source of microphonic noise arose from argon gas bubbles around the coaxial cables caused by boiling of the liquid argon in the bath around the chamber. Vertical aluminium tubes long enough to stay above the liquid argon level were mounted on each feed-through and filled with paraffin wax. In this way the cables were no longer in contact with the liquid argon and at the same time all the connections were mechanically reinforced.

The sense-wire amplifier was of the charge sensitive type and had a rise-time and decay time constant of $1 \mu \mathrm{s}$ and $40 \mu \mathrm{s}$ respectively. Laboratory tests made using a $1 \mu \mathrm{s}$ shaping time gave the following noise characteristics, $<\mathrm{ENC}>=330$ el for $0 \mathrm{pF}$ input capacitance and a slope of $2.26 \mathrm{el} / \mathrm{pF}$.

Tests made in actual working conditions without using any shaping circuit gave the following results, $\langle\mathrm{ENC}\rangle=600 \mathrm{el}$ for $\mathrm{C}_{\mathrm{in}}=0 \mathrm{pF}$ and $\langle\mathrm{ENC}\rangle=1300 \mathrm{el}$ for $\mathrm{C}_{\mathrm{in}}=$ $120 \mathrm{pF}$.

This noise figure corresponds to a signal to noise ratio of 4.5 for a signal of 6000 electrons. We did not use any shaping circuit because of the triangular shape of the 
induced signal. A shaper would have caused the loss of the rise time information of the induced signal. The gain of the amplifier was $56 \mathrm{mV} / \mathrm{fC}$. A test pulse could be injected through a $1 \mathrm{pF}$ test capacitor to determine the calibration factor. We used a test pulse of amplitude $1.6 \mathrm{mV}$ giving an input charge of 10000 electrons.

The anode was also connected to an amplifier and this could be read out to obtain the total charge deposited on the wires. We used the same amplifier as for the sensewires but with a longer decay time of $80 \mu$ s and reduced gain to prevent saturation of the signals.

The sense-wires amplifiers were housed in two boxes, each box containing 16 amplifiers. The anode amplifier was in an individual box together with its high voltage ( $\mathrm{V}_{\mathrm{a}}$ in Fig. 6) decoupling capacitor and the biasing resistor. The three boxes were in turn placed in a Faraday cage and a supplementary shield was put around the signal cables. The cage was located next to the chamber to minimize cable length.

After the preamplifier stage, the signals were passed on to a digitizer board[17]. The board contained 40 channels, each channel consisted of a line receiver, an 8 bit flash $\mathrm{ADC}$ and two 8 kbyte $\times 8$ memories. The flash ADC digitized continuously at a rate of $5 \mathrm{MHz}$ and stored the information in the first $8 \mathrm{k}$ memory. After a trigger, a delay of $1.6 \mathrm{~ms}$ was generated. At the end of the delay a stop signal was supplied and digitizing ceased. The data were then transferred to the second $8 \mathrm{k}$ memory for reading by the VME system. One could either readout the complete $8 \mathrm{k}$ of memory after the stop signal, or else only part of the memory. When a trigger arrived a test pulse was generated and was digitized along with the event to indicate the start of drift.

The data acquisition system was VME based and was controlled using Macintosh microcomputers[18]. The VME crate was also connected to a $\mu$ Vax II via an interface known as VASCO[19]. The data were transferred to the $\mu$ Vax II where they were recorded on magnetic tape for further analysis.

\section{Results}

A total of about 12000 triggers were recorded at different electric fields and drift distances. At $500 \mathrm{~V} / \mathrm{cm}$ and $200 \mathrm{~V} / \mathrm{cm}$ a total of $\approx 1200$ triggers were taken at each field and drift distance (nominal values of 40,80,120,160,220 $\mathrm{mm}$ ). We also recorded $\approx 14000$ triggers at $500 \mathrm{~V} / \mathrm{cm}$ and $8 \mathrm{~cm}$ drift distance to study the production of deltarays.

\subsection{Pulse height and pulse shape measurements}

It is clearly important to demonstrate that the observed pulses on the sense-wires are well described by the theoretical shapes given in Fig. 3b. Before making any comparisons between the expected pulse shapes and the data we have checked the 
quality of the liquid argon by measuring the electron lifetime using data taken at 500 $\mathrm{V} / \mathrm{cm}$. The total charge deposited in $2 \mathrm{~mm}$ was measured by setting the anode voltage to zero, meaning that the induced signal terminated at the sense-wires. The pulse height as a function of distance is shown in Fig. 7 together with curves for $\tau$ of $1 \mathrm{msec}$ and $10 \mathrm{msec}$, showing that the lifetime is of the order of several milliseconds, as measured in a previous test[9].

We then made fits to the measured pulses using the theoretical pulse shapes. In Fig. 8 are shown a series of pulses for the five drift distances and fields of $200 \mathrm{~V} / \mathrm{cm}$ and $500 \mathrm{~V} / \mathrm{cm}$.

The function $F\left(t_{i}\right)$ used to fit the data is a combination of a linear background and the theoretical pulse shape, namely

$$
F\left(t_{i}\right)=A t_{i}+B+H f_{t h}\left(t_{i}+T\right)
$$

where $t_{i}$ is the time corresponding to bin $\mathrm{i}, \mathrm{A}$ and $\mathrm{B}$ are parameters describing the background, $H$ is a scale factor that normalizes the theoretical pulse shape $f_{t h}$ to the measured pulse and $\mathrm{T}$ is a time offset measured from the trigger time. In order to reduce the effects of the electronic noise on the pulse shape, the pulses at a given field and drift distance have been averaged together. The theoretical shape also included the amplifier response. The resulting fits are shown as the solid curves in Fig. 8. The agreement between the measured and fitted pulses is very good.

The measured amplitudes in number of electrons are given in Table 1. The pulse height at $200 \mathrm{~V} / \mathrm{cm}$ is lower than that at $500 \mathrm{~V} / \mathrm{cm}$ due to the increased recombination of the electron-ion pairs that takes place at the lower field. In Fig. 9 we plot the measured amplitudes as a function of distance.

The amplitudes are lower than expected for two reasons: 1) the induced charge never reaches $100 \%$, the mean value being $75 \%, 2$ ) due to the rise-time of the amplifier the pulse height is reduced by $20 \%$. In addition, the amplitude decreases faster with distance than would be expected by the measured lifetime. This decrease is due to the diffusion of the $2 \mathrm{~mm}$ electron track. There are two types of diffusion, lateral and longitudinal. The lateral diffusion is not expected to play any role here, as any movement of electrons towards the adjacent sense-wire gap will be compensated by electrons moving in the opposite direction. As the electrons drift across the grid/sensewire gap there may be some broadening of the electron track but the effect is expected to be negligible. However, the effects due to longitudinal diffusion will have a more marked effect on the track. The longitudinal spread of the electron track is given by

$$
\sigma=\sqrt{2 \mathrm{Dt}}
$$


where $\mathrm{D}$ is the diffusion coefficient and $\mathrm{t}$ is time in seconds. For thermal electrons we have the Einstein-Debye relationship, namely

$$
\mathrm{D}=\frac{\mathrm{k}_{\mathrm{B}} \mathrm{T}}{\mathrm{e}} \mu
$$

where $\mu$ is the electron mobility, $\mathrm{T}$ is the temperature, $\mathrm{k}_{\mathrm{B}}$ is Boltzmann's constant and $\mathrm{e}$ is the electronic charge. Making the relevant substitutions in equation 5 with $\mathrm{T}=87 \mathrm{~K}$ gives $\mathrm{D}=4 \mathrm{~cm}^{2} \mathrm{~s}^{-1}$. Electrons in liquid argon are thermal up to electric fields of $\approx 200$ $\mathrm{V} / \mathrm{cm}$. At higher fields the electrons gain more energy from the field than they lose in collisions with the argon atoms and so become non-thermal and hence equation 5 no longer applies. However, for fields $\leq 1 \mathrm{kV} \mathrm{cm}{ }^{-1}$ the diffusion coefficient has a constant value of $\approx 4 \mathrm{~cm}^{2} \mathrm{~s}^{-1}$, the low field value. Longitudinal diffusion spreads out the electron track in time so for a track segment which is parallel to the wire-plane the electrons no longer arrive all at the same time. This leads to a broadening of the pulse on the sense-wires, and therefore a reduced pulse height (charge must be conserved). Although this time spread is small, $\leq 0.2 \mu \mathrm{s}$ at $22 \mathrm{~cm}$ drift and $500 \mathrm{~V} / \mathrm{cm}$, the signal is very narrow, hence even this small spread has a noticeable effect. This spread has been included in the theoretical pulse shapes used to fit the measured pulses. The curves in Fig. 9 are obtained from the fitted curves in Fig. 8 and include an electron lifetime of $10 \mathrm{msec}$.

\subsection{Measurement of the electron drift velocity}

Using the data taken at different drift distances we have measured the drift velocity for electric fields $E=500 \mathrm{~V} / \mathrm{cm}$ and $\mathrm{E}=200 \mathrm{~V} / \mathrm{cm}$. For each electric field and drift distance the position in time of the signal is determined and then averaged over many signals. This average time is plotted as a function of the drift distance in Fig. 10 for the two electric fields. We find that due to uncertainties in the measurement of the drift distance there is an offset of $0.8 \mathrm{~cm}$ meaning that the $4 \mathrm{~cm}$ drift distance is actually $3.2 \mathrm{~cm}$. This offset has been corrected for when plotting the data in Fig. 10. The curves are a straight line fit of the form

$$
d=v_{d} t_{d}
$$

and yield

$$
\begin{array}{ll}
\mathrm{v}_{\mathrm{d}}=1.55 \mathrm{~mm} / \mu \mathrm{s} & \text { at } 500 \mathrm{~V} / \mathrm{cm} \\
\mathrm{v}_{\mathrm{d}}=0.89 \mathrm{~mm} / \mu \mathrm{s} & \text { at } 200 \mathrm{~V} / \mathrm{cm}
\end{array}
$$

These values compare well with values determined previously by us[9] and by other authors[7,20-24]. 


\subsection{Observation and measurement of delta-rays}

One of the purposes of the ICARUS experiment[8] is to detect and measure the recoil electrons from the interaction of solar neutrinos within the detector volume. It is therefore important to be able to identify and measure electrons with energies of a few $\mathrm{MeV}$.

A particle travelling through matter leaves a trail of electron-ion pairs along its path. If the liberated electrons have sufficient energy then they may form an independent track, creating a further series of electron-ion pairs. These electron tracks are referred to as delta-rays. The kinetic energies of the recoil electrons from the interactions of neutrinos from the ${ }^{8} \mathrm{~B}$ cycle in the sun are in the range $0-13.5 \mathrm{MeV}$, similar to the energies of the delta-rays that will be contained in the chamber.

An incident particle of kinetic energy $T$ can lose energy up to a value $T_{\max }$, where

$$
T_{\max }=\frac{2 m_{e} \gamma^{2} \beta^{2}}{\left(1+2 \gamma\left(m_{e} / m\right)+\left(m_{e} / m\right)^{2}\right)}
$$

with $m_{e}=$ electron rest mass, $\beta=v / c$ velocity of incident particle, $m=$ mass of incident particle. For a pion of momentum $5 \mathrm{GeV} / \mathrm{c}$ this maximum energy loss is $1 \mathrm{GeV}$. The number of electrons of energy $T \ll T_{\max }$ produced per cm per unit energy is[25]

$$
\frac{\mathrm{d}^{2} \mathrm{~N}}{\mathrm{dTdx}}=\frac{1}{2} \mathrm{D}\left(\frac{Z_{\text {med }}}{A_{\text {med }}}\right)\left(\frac{Z_{\text {inc }}}{\beta}\right) \rho_{\text {med }} \frac{1}{T^{2}}
$$

where $\mathrm{D}=0.307 \mathrm{MeV} \mathrm{cm} \mathrm{g}^{-1}, \mathrm{Z}_{\text {med }}$ and $\mathrm{A}_{\text {med }}$ are the atomic number and mass respectively (which for argon are 18 and 40 ), $\rho_{\text {med }}$ is the density of the medium (1.4 $\mathrm{g} / \mathrm{cm}^{3}$ for liquid argon) and $Z_{\text {inc }}$ is the charge of the incident particle. For $\mathrm{T} \approx \mathrm{T}_{\max }$ there are corrections to equation 8 arising from the spin of the incident particle. Integrating equation 8 over $x(=6.2 \mathrm{~cm})$ gives

$$
\frac{\mathrm{dN}}{\mathrm{dT}}=\frac{0.6}{\mathrm{~T}^{2}}
$$

hence the number of delta-rays with energies greater than $T_{\min }$ produced per pion is

$$
\mathrm{N}\left(\mathrm{T}_{\min }<\mathrm{T}<\mathrm{T}_{\max }\right)=0.6\left(\frac{1}{\mathrm{~T}_{\min }}-\frac{1}{\mathrm{~T}_{\max }}\right)
$$

For $\mathrm{T} \ll \mathrm{T}_{\max }$ the number of delta-rays per pion is given by $0.6 / \mathrm{T}_{\min }$, hence for $\mathrm{T}_{\min }>$ $1 \mathrm{MeV}, 60 \%$ of the events should contain a delta-ray.

The data sample used for this measurement was 14276 events taken at $500 \mathrm{~V} / \mathrm{cm}$ and $8 \mathrm{~cm}$ drift distance. An event was considered to contain a delta-ray candidate if there was a second track, connected to the main track of the pion, with at least two 
pulses separated from the main track by at least $4 \mathrm{~mm}$. For $500 \mathrm{~V} / \mathrm{cm}$ the drift velocity was $1.55 \mathrm{~mm} \mathrm{\mu s}^{-1}$ so $4 \mathrm{~mm}$ separation corresponded to 13 time bins where one time bin was $200 \mathrm{~ns}$. Four typical events selected in this way are shown in Fig. 11. The pion track occurs between 70 and $80 \mu \mathrm{s}$, as expected for the $8 \mathrm{~cm}$ drift distance. The track at $27 \mu$ s is a test pulse (generated with the trigger) which indicates the start of drift. The delta-rays appear as distinct tracks at an angle to the pion track. The direction of the pion is from wire 31 to wire 1 . To obtain the energy of the delta-ray we sum up the energies of the individual pulses satisfying the above criteria. The presence of only one space co-ordinate makes it difficult to extract any reliable measure of the angle of emission of the electron although the energy can still be determined.

It is often difficult to identify the start of the delta-ray as it may be superimposed on the pion track. Also, multiple Coulomb scattering can considerably distort the path of the electron. Hence the requirement on the pulse separation means that we do not reconstruct all the energy deposited by the delta-ray. To study this energy loss we have used a Monte Carlo to generate events containing delta-rays of a given energy and have simulated the effects of the multiple scattering. The events are then passed through our drift chamber simulation program. Two such events are shown in Fig. 12, generated with energies of $10 \mathrm{MeV}$ (Fig. 12a) and $15 \mathrm{MeV}$ (Fig. 12b) respectively. We then apply the same selection criteria to our Monte Cario events and compute the visible energy for each event and compare it to the generated energy. This is repeated for many events and the mean visible energy is calculated. Fig. 13 shows the curve obtained for generated energies from $1.5 \mathrm{MeV}$ to $23 \mathrm{MeV}$. The percentage of energy seen is not a constant but varies from $40 \%$ for a generated energy of $5 \mathrm{MeV}$ to $63 \%$ for a generated energy of $23 \mathrm{MeV}$. We then use this curve to correct the data.

Using the Monte Carlo we can also determine the acceptance of the events as a function of electron energy. Several factors contribute to reduce the acceptance to less than $100 \%$. Firstly low energy electrons are difficult to identify as they may only contribute one pulse with more than $4 \mathrm{~mm}$ separation from the pion track. Secondly, the single wire plane means that for certain angles of emission of the electron it will not be seen as a separate track. Problem configurations are 1) electrons in the same vertical plane as the pion and 2) electrons emitted parallel to the sense-wires. In the first case the electron track will be superimposed on that of the pion and in the second case it will deposit all of its charge on one wire. Thirdly, due to the limited fiducial volume, high energy electrons will not be contained in the chamber. For this reason we restrict our sample to those events where there are no pulses on wires 1 or 31 that contribute to the delta-ray. In Fig. 14 we show the acceptance curve for the selection criteria. For an electron energy of $5 \mathrm{MeV}$ the acceptance is $37 \%$ falling to $16 \%$ at $20 \mathrm{MeV}$, due to electrons not contained in the chamber. For energies below $3 \mathrm{MeV}$ the acceptance is 
less than $1 \%$ and between $1.5 \mathrm{MeV}$ and $3 \mathrm{MeV}$ it falls by two orders of magnitude, making the acceptance in this region rather uncertain.

The fully corrected data are shown in Fig. 15 together with the theoretical prediction (error bars are statistical only). The curve has been obtained from equation 9 and includes the acceptance from Fig. 14 and the total number of events, $\mathrm{N}_{\text {tor. }}$. Above $5 \mathrm{MeV}$ the curve gives a good description of the data. In the peak the Monte Carlo predicts less events than are actually observed but as has been noticed the acceptance in this region is falling very fast and is probably less reliable. In addition there is a systematic error on the energy scale of $+10 \%$ due to uncertainties in the absolute calibration.

Our final data sample contains 880 events. The expected number of events $\mathrm{N}$ can be obtained from equation 9

$$
\mathrm{N}=\mathrm{N}_{\mathrm{tot}} \times 0.6 \int_{\mathrm{T}_{\max }}^{\mathrm{T}_{\min }} \mathrm{A}(\mathrm{T}) \frac{1}{\mathrm{~T}^{2}} \mathrm{dT}
$$

where $A(T)$ is the acceptance as a function of energy. The limited acceptance reduces the expected percentage of events containing delta-rays from $60 \%$ to about $6 \%$. This gives the expected number of events as 892 which is in good agreement with the observed number of events.

\section{Conclusions}

We have tested a liquid argon TPC containing a sense-wire plane to obtain nondestructive detection of the electron image of ionizing events. The novel wire configuration was based on the principle of electrostatic focussing. The measured pulse shapes at both $200 \mathrm{~V} / \mathrm{cm}$ and $500 \mathrm{~V} / \mathrm{cm}$ were in very good agreement with the expected shapes, calculated taking into account the electron lifetime, the response of the electronics and the longitudinal diffusion of the $2 \mathrm{~mm}$ electron cloud. The measured electron drift velocity was in good agreement with the results of other workers as well as with our previous measurements.

In addition we have selected a sample of delta-rays in order to study the behaviour of low energy electrons in the liquid argon. We find that for electron energies greater that $5 \mathrm{MeV}$ the measured and predicted spectrums agree very well after the acceptance and energy losses have been corrected for, demonstrating the feasibility of recognising low energy electrons.

We have demonstrated that the idea of electron imaging using grids of wires works as expected. We obtained clear signals above the electronic noise (signal-tonoise ratio of 4.5 ) for a signal of 6000 electrons. This is clearly an important step in 
the realisation of the ICARUS experiment and we now plan to construct a full scale prototype based on this wire design.

\section{Acknowledgements}

We gratefully acknowledge the support of the Istituto Nazionale di Fisica Nucleare in funding this work. Thanks are also due to the following people who have helped in the preparation of the work described here: L. Bassi, M. Benvenuto, A. Bochaton, M. Cicia, C. Detraz, A. Galvagni, K. Morgan, J. Oliver, R. Pavanello and R. Truhan. 


\section{REFERENCES}

[1] C. Rubbia, CERN-EP Internal Report 77-8 (1977), unpublished.

[2] H.H. Chen and J.F. Lathrop, Nucl. Instr. and Methods 150, (1978) 585.

[3] H.H. Chen and P.J. Doe, IEEE Trans. Nucl. Sci. NS-28, (1981) 454.

[4] P.J. Doe, H.J. Mahler and H.H. Chen, Nucl. Instr. and Methods 199, (1982) 639.

[5] K. Dieters et al., Nucl. Instr. and Methods 180, (1981) 45.

[6] E. Gatti et al., IEEE Trans. Nucl. Sci. NS-26, No. 2 (1979) 2910.

[7] E. Aprile, K.L. Giboni and C. Rubbia, Nucl. Instr. and Methods A241, (1985) 62.

[8] ICARUS proposal, INFN/AE-85/7 FRASCATI (1985).

ICARUS I: an optimized, real time detector of solar neutrinos. Proposal for the ICARUS experiment phase 1, CERN-Frascati-L'Aquila-Padova-UCLA Collaboration (1988), unpublished.

[9] E. Buckley et al., CERN-EP/88-120 (1988), to be published in Nucl. Instr. and Methods.

[10] Messer Griesheim GmbH, Oxisorb Gas purifying system.

[11] A. Gonidec et al., CERN-EP/88-36 (1988), submitted to Nucl. Instr. and Methods.

M. Albrow et al., Nucl. Instr. and Methods A265, (1988) 303.

[12] O. Bunemann, T.E. Cranshaw and J.H. Harvey, Can. J. Res. 27, (1949) 191.

[13] This procedure was originally developed for the cleaning of the stainless steel vacuum elements of the CERN Intersecting Storage Rings. See A.G.

Mathewson, CERN-ISR-VA 74-10 and 74-30, unpublished.

M-H. Achard,R. Calder and A.G. Mathewson, Vacuum 29, No. 2 (1978)

53.

[14] MACOR is a machineable glass ceramic made by CORNING.

[15] ConFlat is a trademark of VARIAN, USA.

[16] Y. Strausser, 'Review of Outgassing Results', technical report VR-51, Vacuum Division, Varian Associates, (1968).

[17] The digitizer board was designed and constructed by J. Oliver, Harvard University, USA.

[18] Macintosh is a trademark of Apple Computer Inc, USA.

[19] VASCO is an interface between $\mu$ Vax and VME developed jointly at the Universities of Padova and Pavia, Italy.

[20] K. Yoshino, U. Sowada and W.F. Schmidt, Phys. Rev. A14, (1976) 438. 
[21] E. Shiburama et al., Nucl. Instr. and Methods 131, (1975) 131.

[22] L.S. Miller, S. Howe and W.E. Spear, Phys. Rev. 166, (1968) 871.

[23] B. Halpern, J. Lekner, S. Rice and R. Gomer, Phys. Rev. 156, (1967) 351.

[24] E.M. Guschchin, A.A. Kruglov and I.M. Obodovskii, Zh. Eksp. Teor. Fiz. 82, (1982) 1114.

[25] 'Review of Particle Properties', Phys. Lett. 170B (1986). 


\section{FIGURE CAPTIONS}

Fig. 1 The wire configuration used in the chamber described in the text. The dimensions are $W=2 \mathrm{~mm}, \mathrm{~d}=2 \mathrm{~mm}, \mathrm{~s}=0.5 \mathrm{~mm}, \mathrm{R}$ (grid wire) $=50 \mu \mathrm{m}$ and $\mathrm{R}$ (sense wire) $=25 \mu \mathrm{m}$.

Fig. 2 The electric field map including the equipotential lines for the wire configuration in Fig. 1 and the ratio of fields of $E_{1}: E_{2}: E_{3} \equiv 1: 5: 5$.

Fig. 3 The induced signals as the fraction of the total charge deposited between the grid wires ( $2 \mathrm{~mm}$ ). a) Sum of the induced signal on both wires as a function of the position of the electron between the wires between the wires. Points A,B, C, D, E and F are as shown. b) Sum of the induced signal on both wires as a function of time for different electron trajectories. Points A, B, C, D, E and $F$ are as shown.

Fig. 4 A cross-section through the chamber.

Fig. 5 A schematic of the experimental set-up and readout system.

Fig. 6 A schematic of the front-end electronics showing the connections to the chamber.

Fig. 7 The pulse height (in arbitrary units) for different distances at $500 \mathrm{~V} / \mathrm{cm}$ using the chamber as a normal gridded ionization chamber together with curves for 1 msec and 10 msec.

Fig. 8 A series of ten pulses taken at different drift distances for a) $200 \mathrm{~V} / \mathrm{cm}$ and b) $500 \mathrm{~V} / \mathrm{cm}$. The superimposed curves are the results of the fits described in the text.

Fig. 9 The pulse height in number of electrons as a function of the drift distance for $200 \mathrm{~V} / \mathrm{cm}$ and $500 \mathrm{~V} / \mathrm{cm}$. The curves are derived from values of the best fit to each of the pulses in Fig. 8. 
Fig. 10 The drift time for $200 \mathrm{~V} / \mathrm{cm}$ (open circles) and $500 \mathrm{~V} / \mathrm{cm}$ (full circles) as a function of drift distance. The data have been corrected for the $0.8 \mathrm{~cm}$ offset described in the text. The curves are a straight line fit of the form $d=v_{d} t_{d}$ and give $v_{d}=0.89 \mathrm{~mm} \mathrm{\mu s}^{-1}$ for $200 \mathrm{~V} / \mathrm{cm}$ and $v_{d}=1.55 \mathrm{~mm} \mu \mathrm{s}^{-1}$ for $500 \mathrm{~V} / \mathrm{cm}$.

Fig. 11 Four typical events containing delta-ray candidates. The track at $27 \mu \mathrm{s}$ is a test pulse and indicates the start of the drift time. The track between $70 \mu \mathrm{s}$ and 80 $\mu \mathrm{s}$ is that of the pion and the delta-ray can be clearly seen emerging at an angle from the main track.

Fig. 12 Two examples of Monte Carlo events containing delta-rays after simulation of the drift chamber. The track at $\approx 27 \mu$ s is a test pulse and indicates the start of the drift time. The track at $82 \mu$ s is the pion track. The generated energies of the delta-rays are a) $10 \mathrm{MeV}$ and b) $15 \mathrm{MeV}$.

Fig. 13 The visible energy of the delta-rays for a given generated electron energy. Only those electrons producing at least two pulses separated from the main track by at least $4 \mathrm{~mm}$ are included in the calculation of the visible energy.

Fig. 14 The acceptance curve as a function of the delta-ray energy for the $4 \mathrm{~mm}$ separation and at least two pulse produced. The decrease at low energy is due to the requirements on pulse separation and number of pulses. The subsequent decrease at higher energies is due to electrons that are not fully contained in our sensitive volume.

Fig. 15 The fully corrected energy spectrum for the delta-rays (error bars are statistical only). The curve is the theoretical prediction from equation 9 after and includes the total number of events $\mathrm{N}_{\text {tot }}$ and the acceptance from Fig. 14. 
Table 1 Number of electrons as a function of drift distance and electric field

\begin{tabular}{l|l|l}
\hline Drift distance (cm) & \multicolumn{2}{|c}{ Number of electrons } \\
& $200 \mathrm{~V} / \mathrm{cm}$ & $500 \mathrm{~V} / \mathrm{cm}$ \\
\hline 4 & $5510 \pm 250$ & $6760 \pm 190$ \\
8 & $5240 \pm 230$ & $6490 \pm 270$ \\
12 & $4980 \pm 210$ & $6200 \pm 240$ \\
16 & $4660 \pm 210$ & $6020 \pm 200$ \\
22 & $4580 \pm 160$ & $5760 \pm 170$ \\
\hline
\end{tabular}




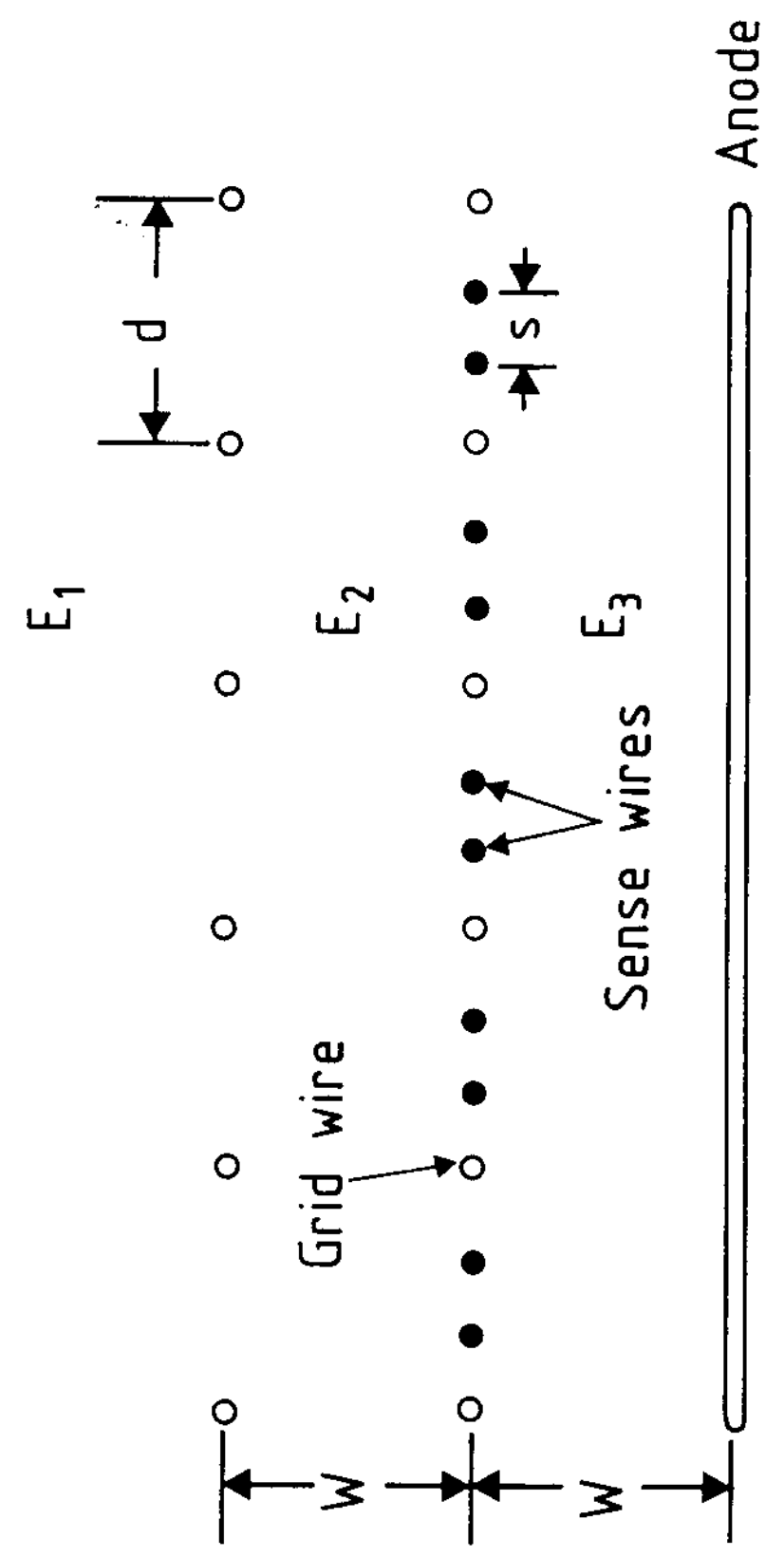

FIG. 1 


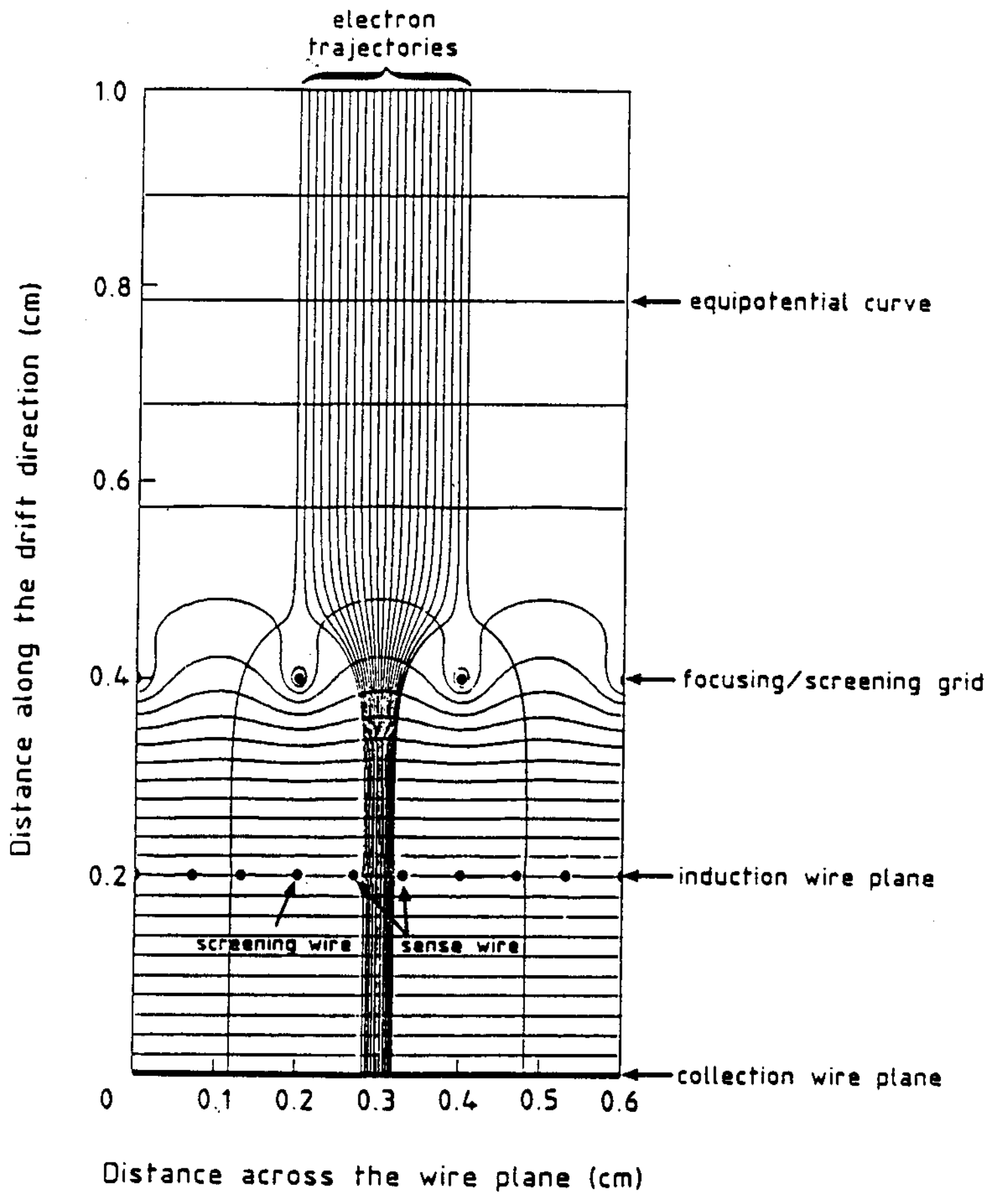

FIG. 2 

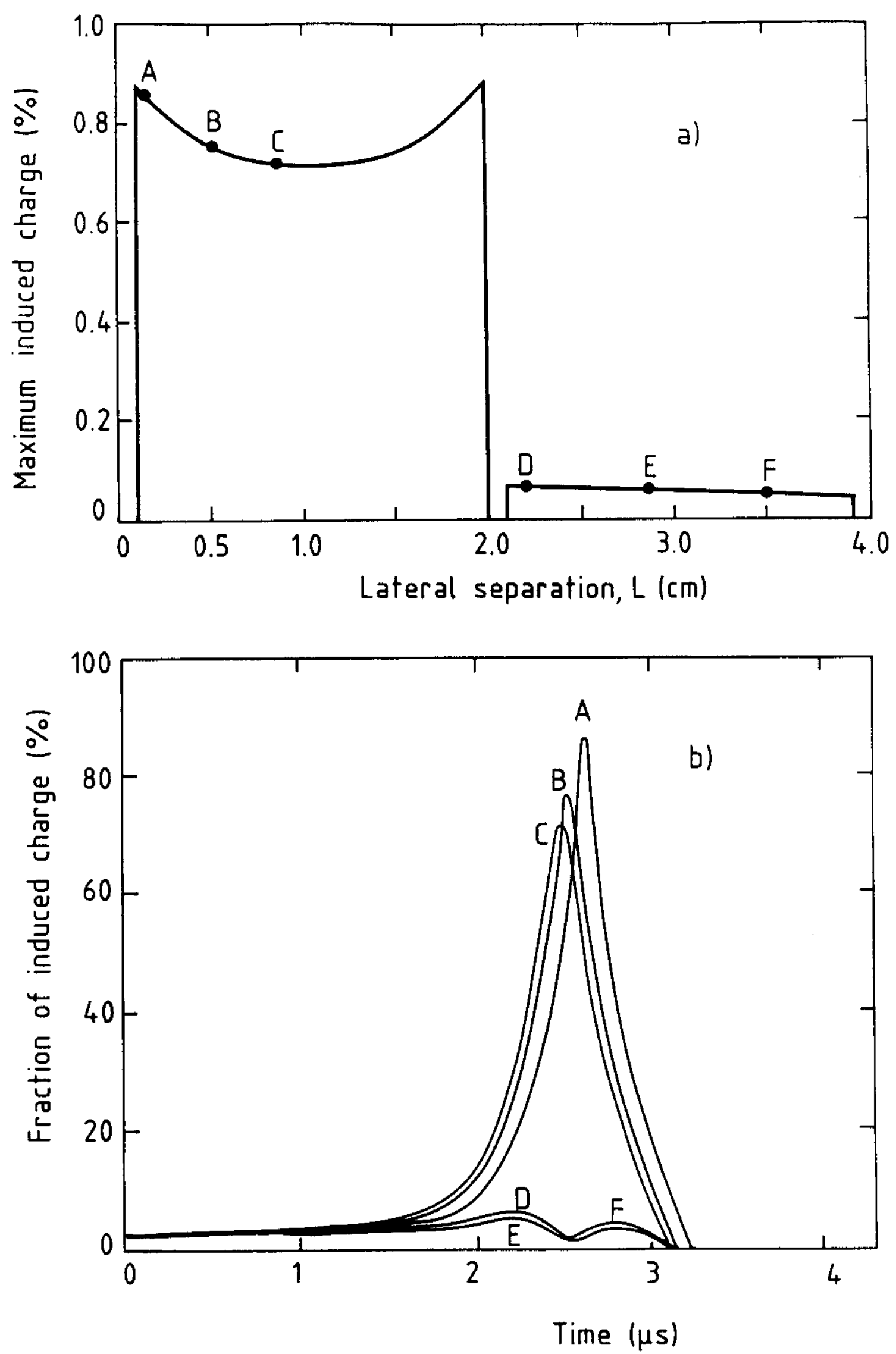

FIG. 3 


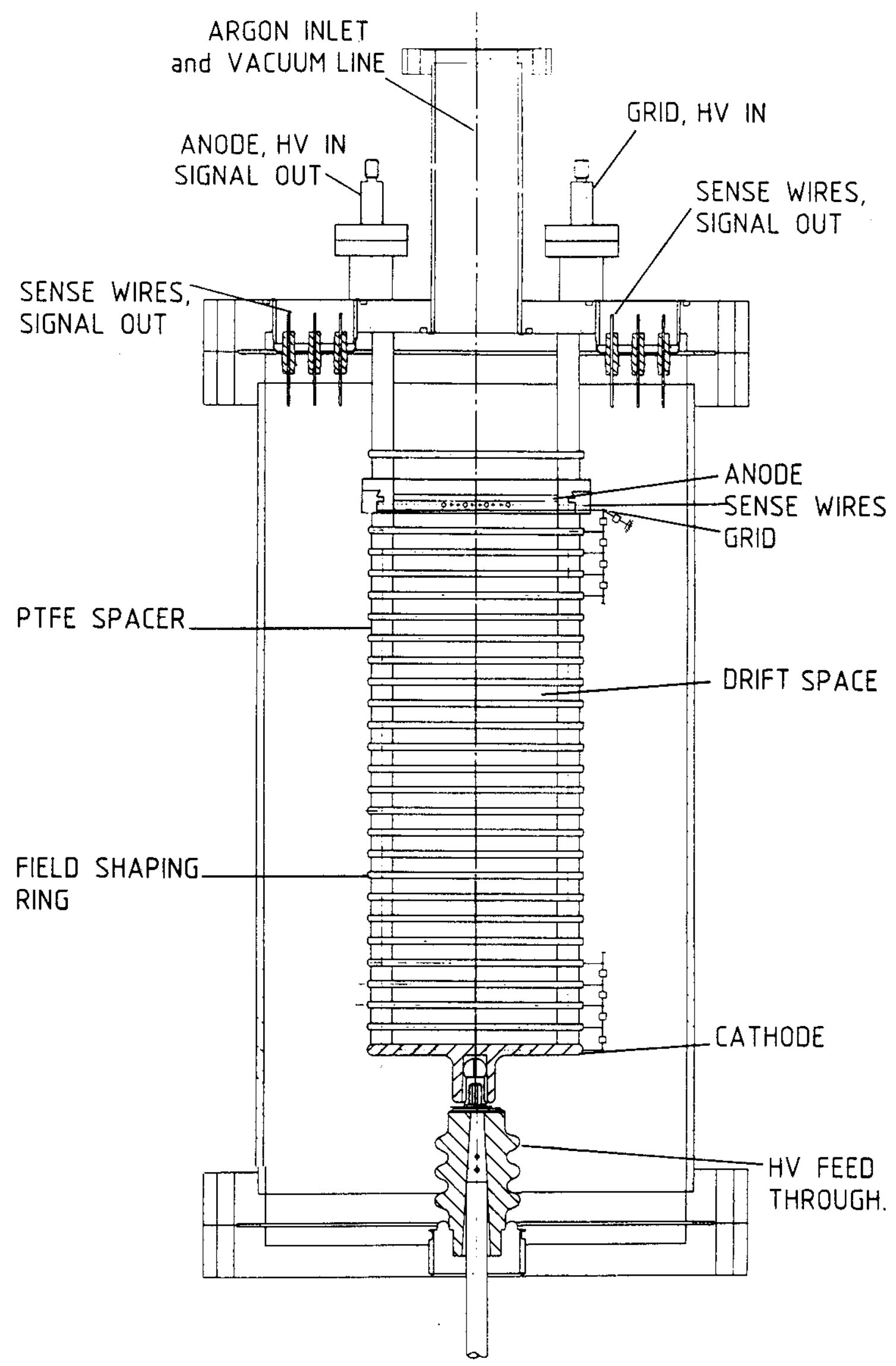




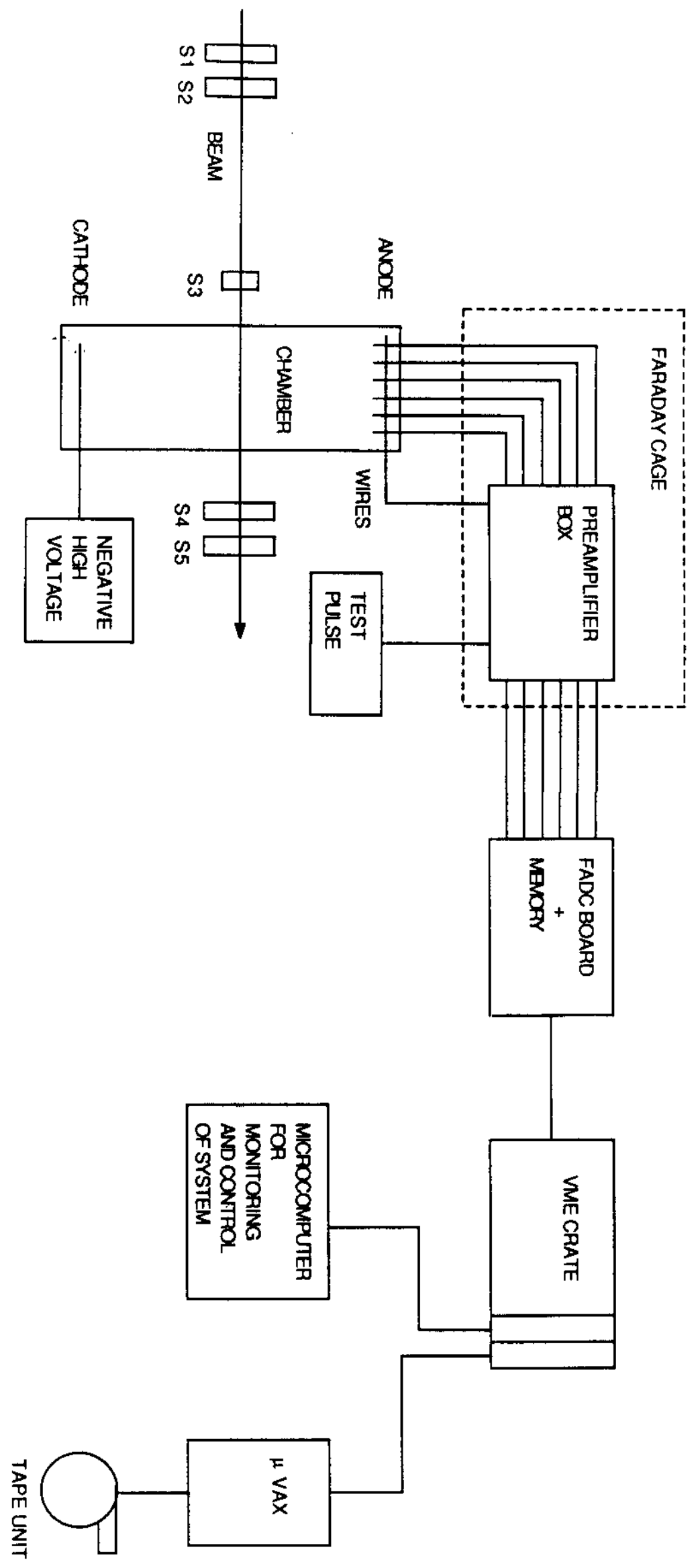

FIG. 5 


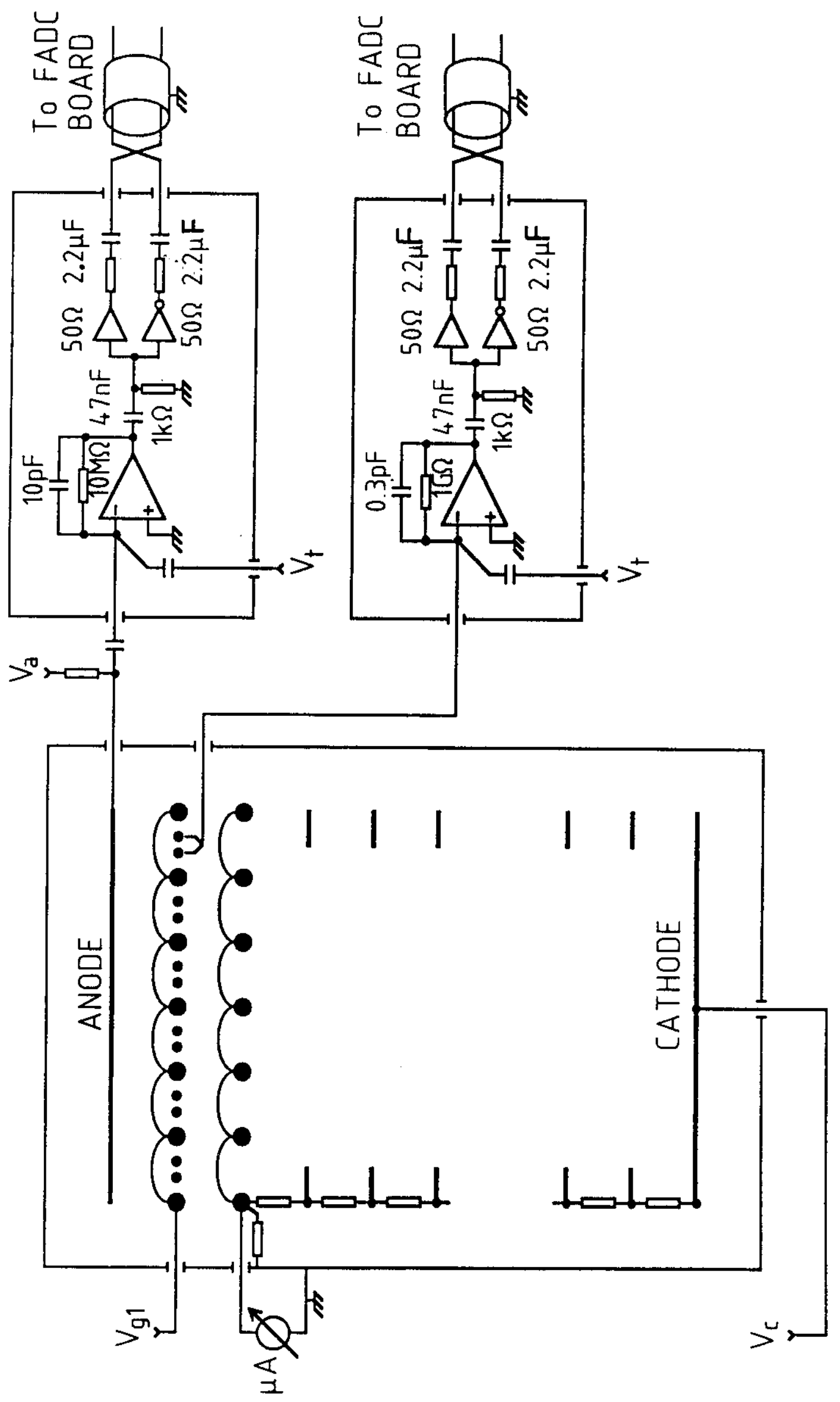

FIG. 6 


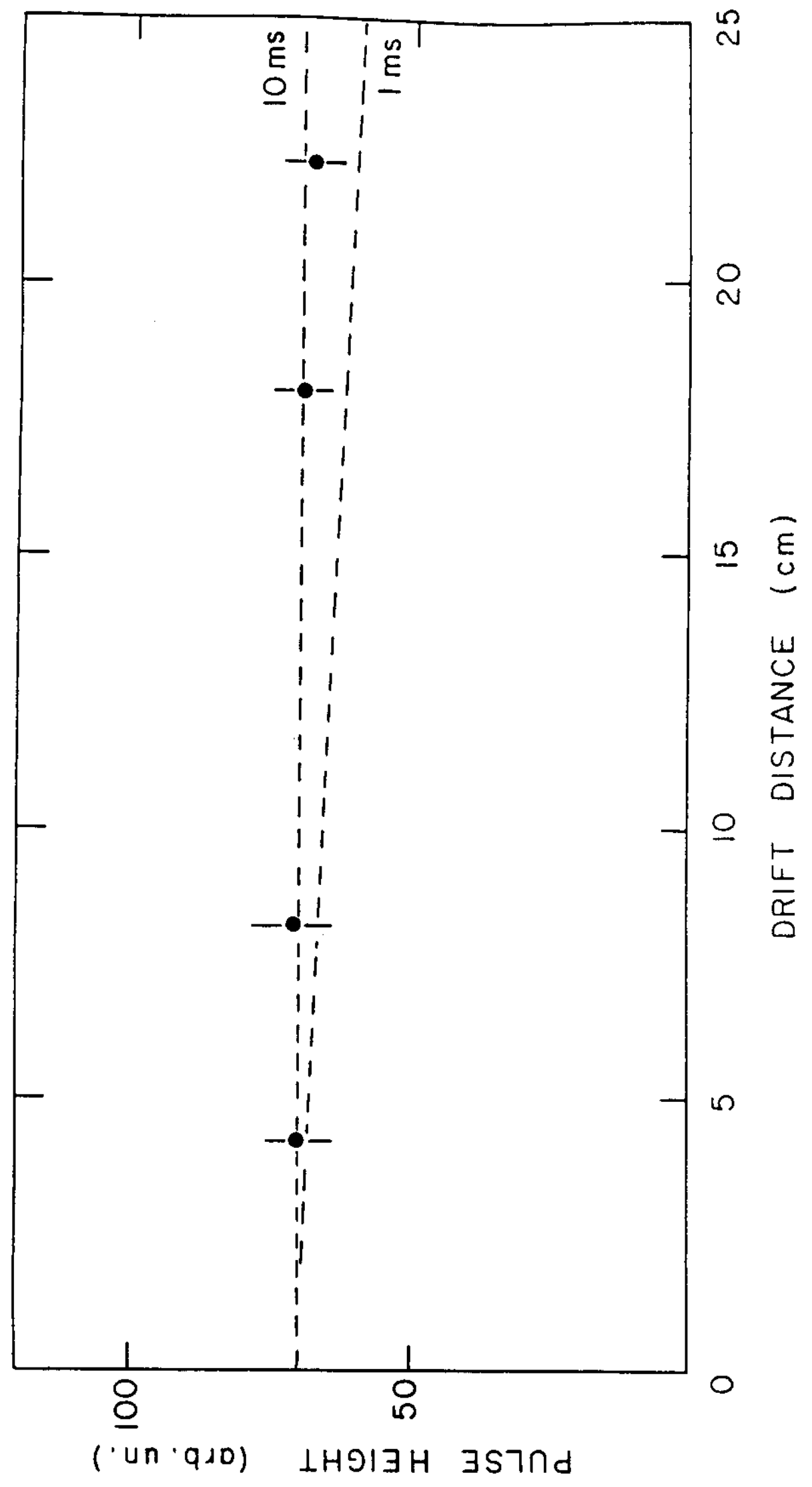

FIG. 7 


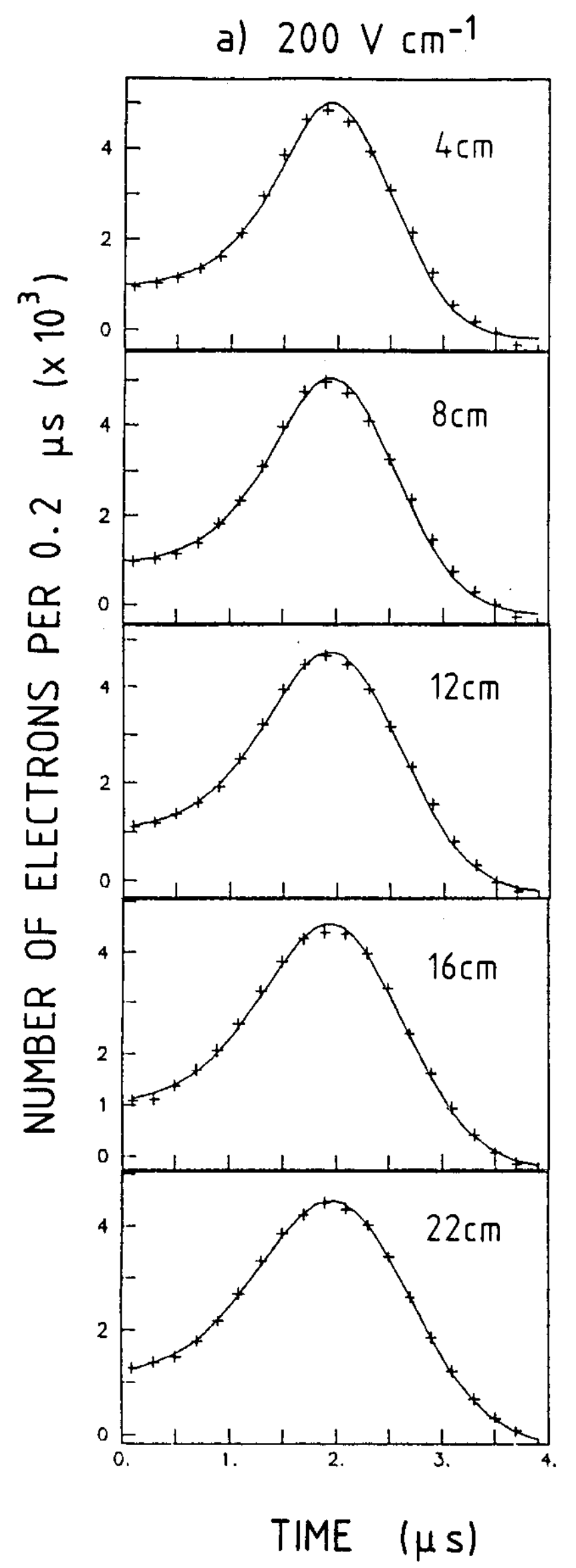

b) $500 \mathrm{~V} \mathrm{~cm}^{-1}$

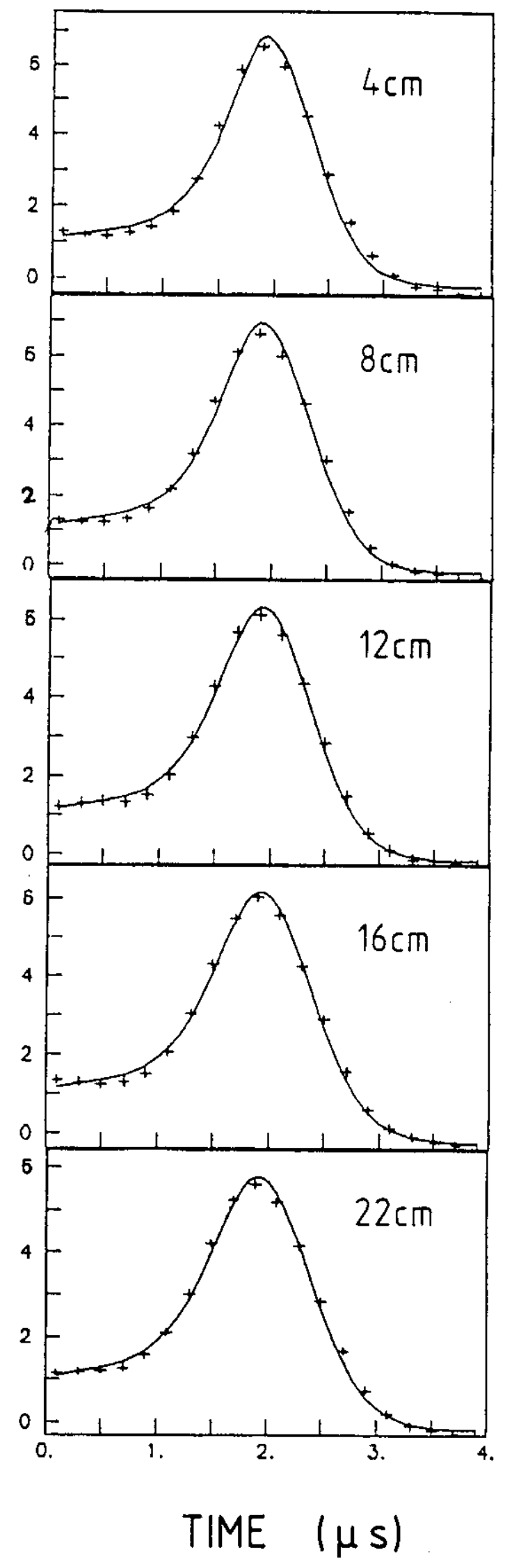

FIG. 8 


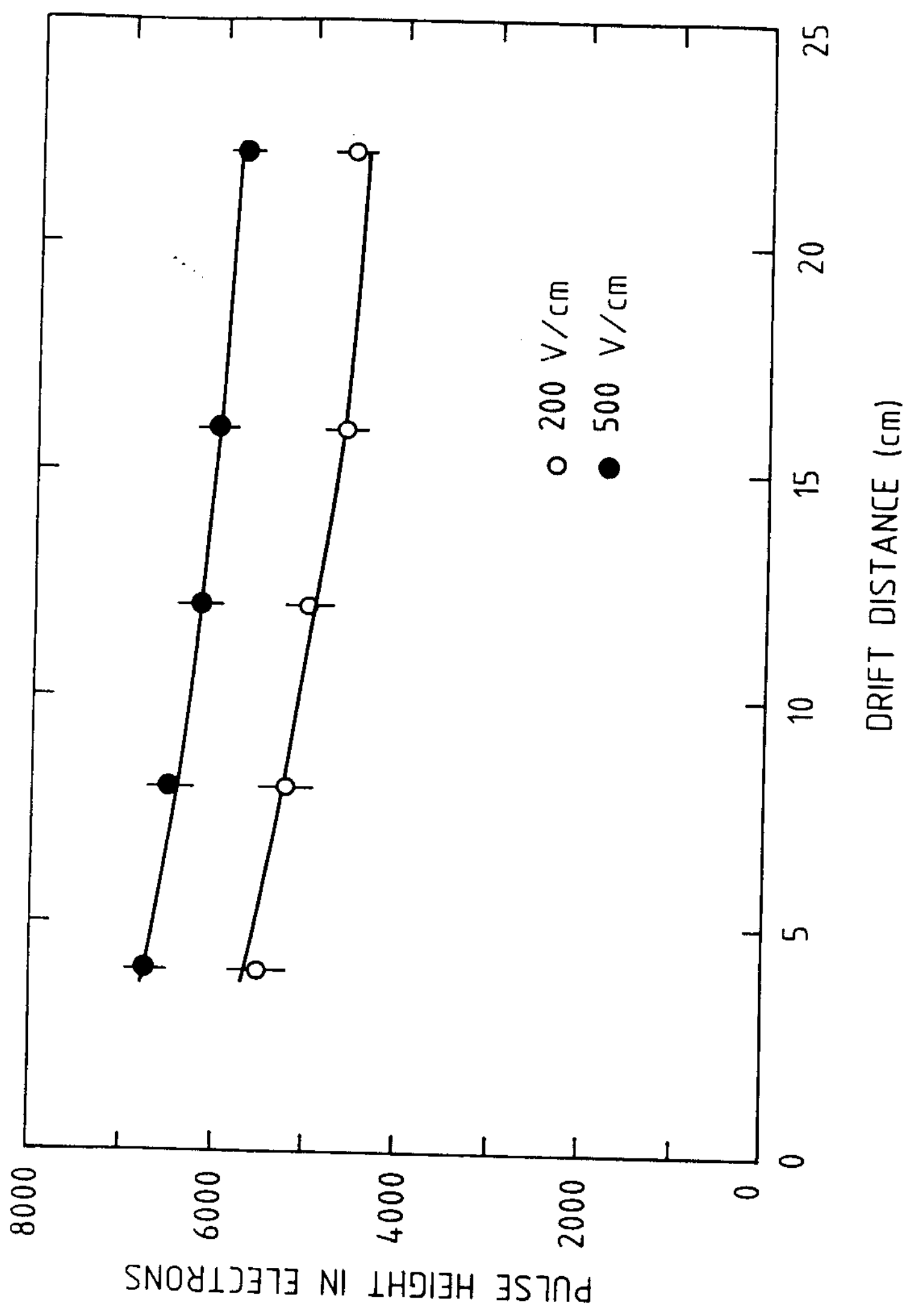

FIG. 9 


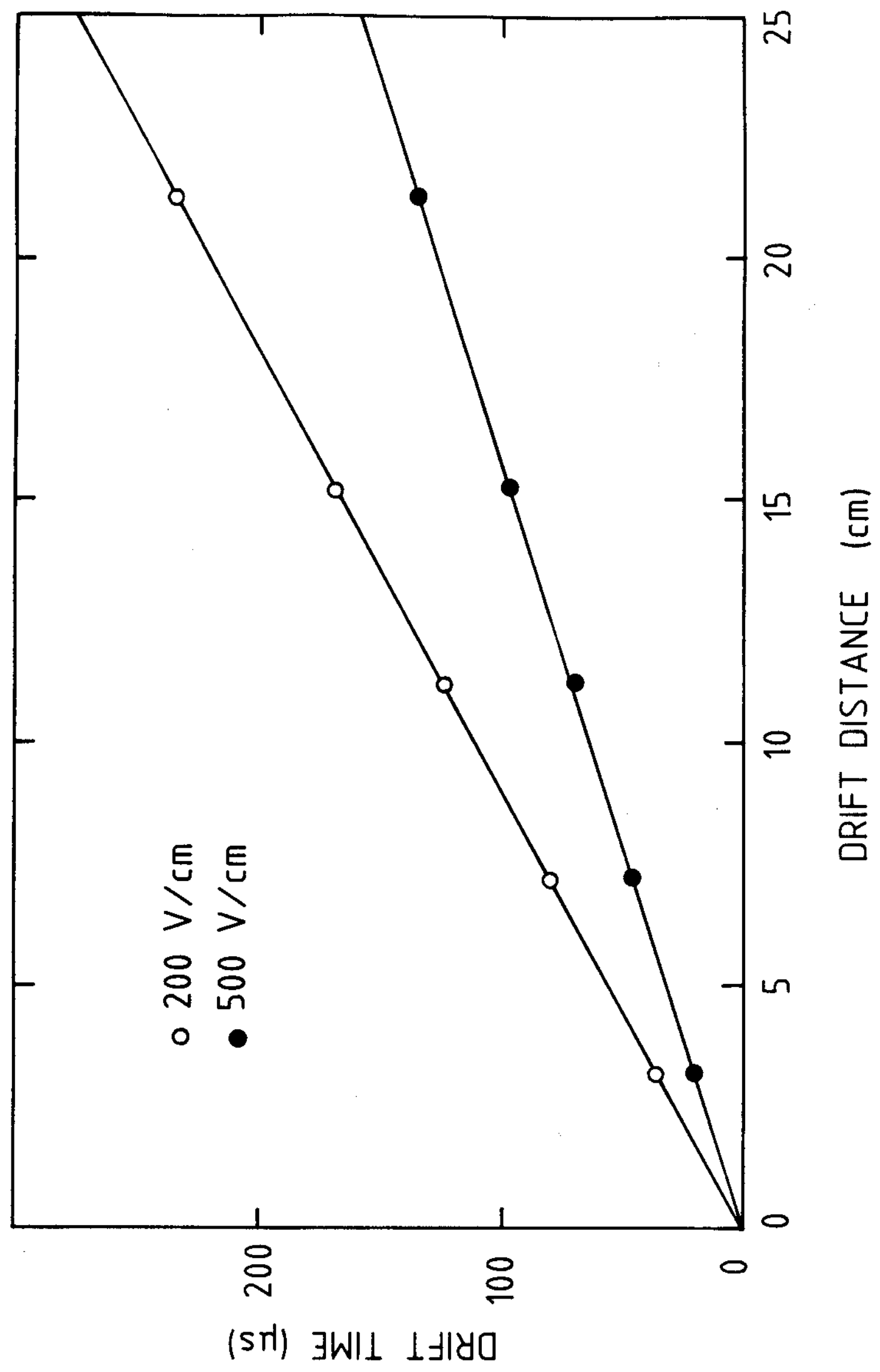

FIG. 10 


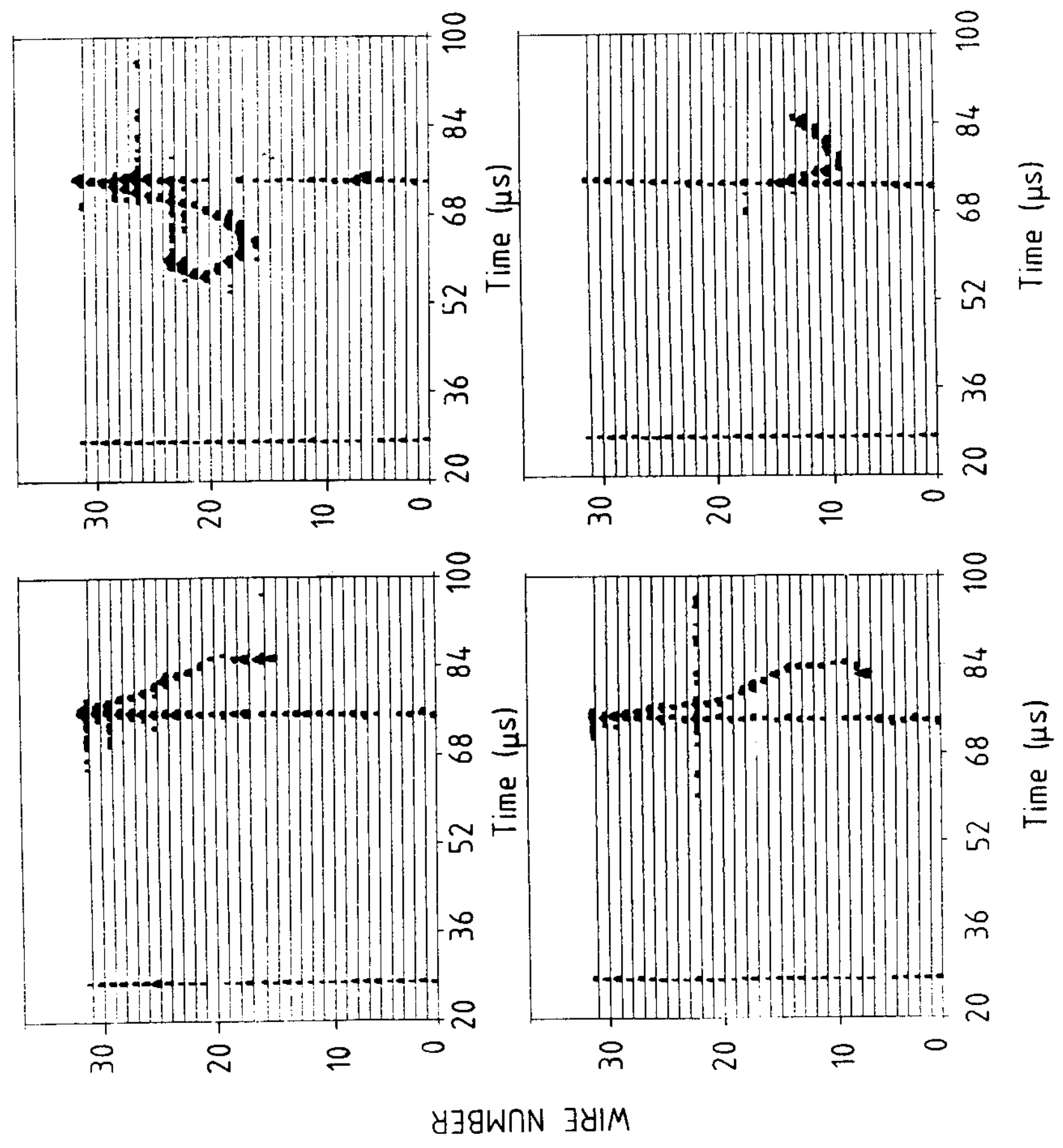

FIG. 11 

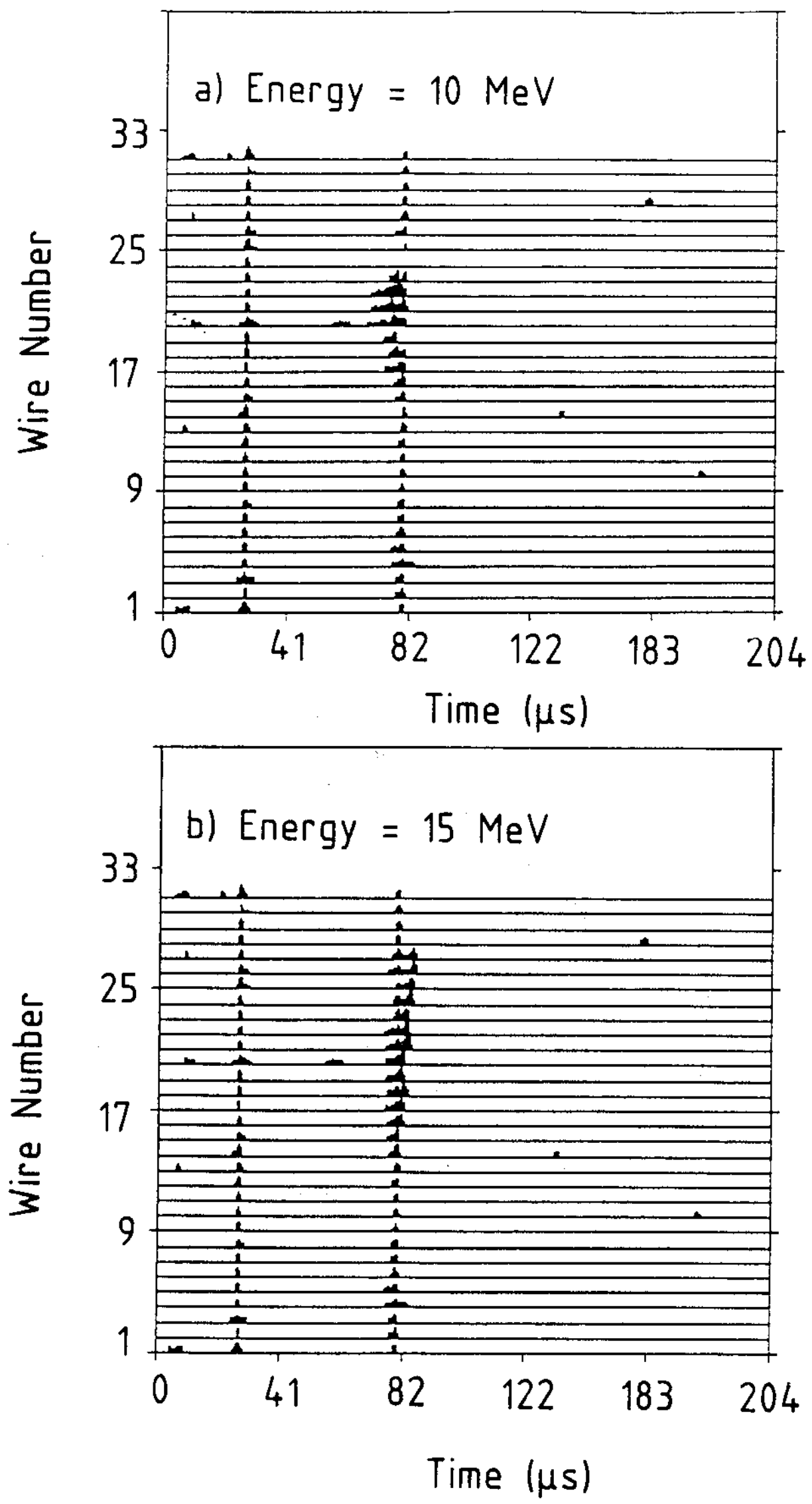

FIG. 12 


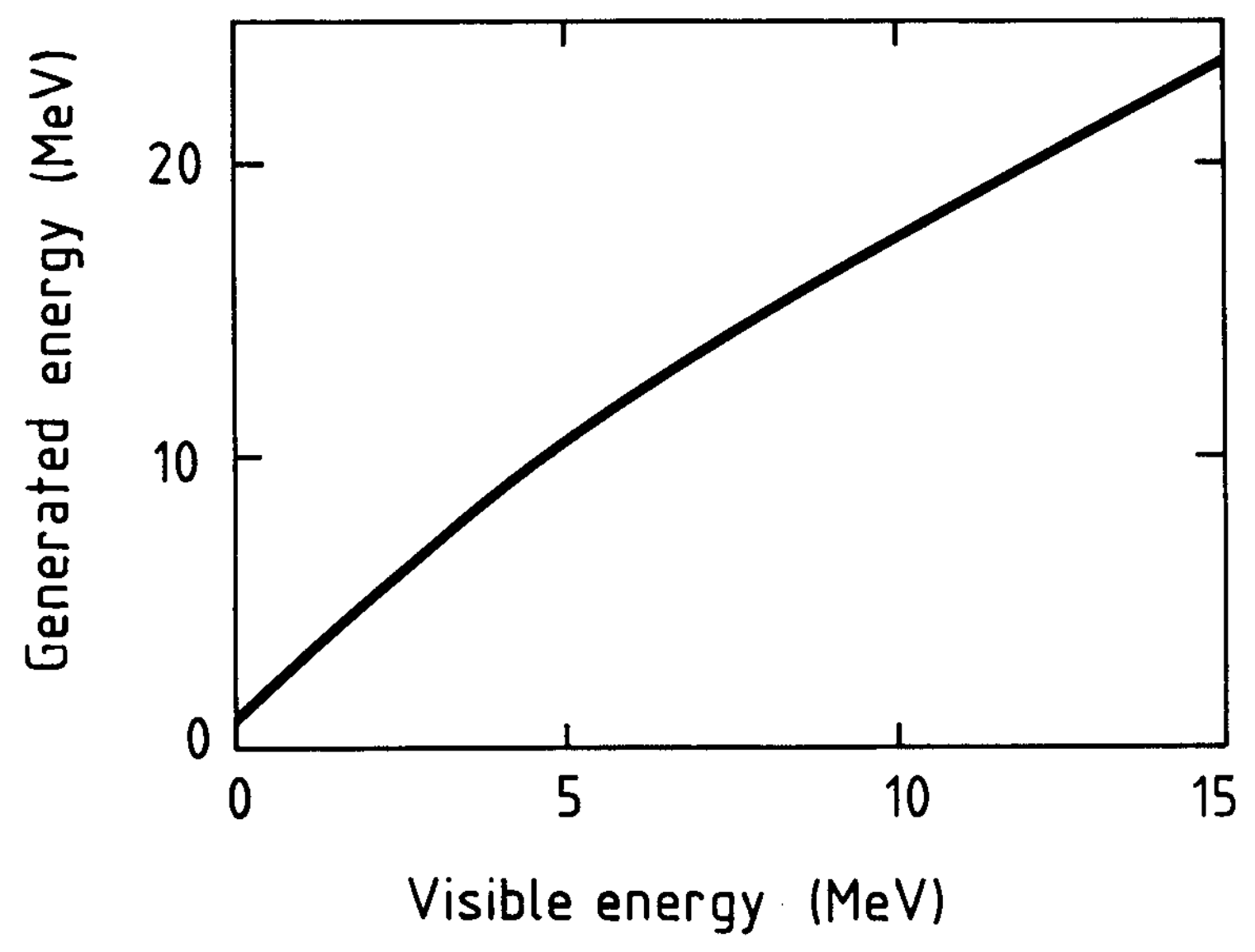

FIG. 13 


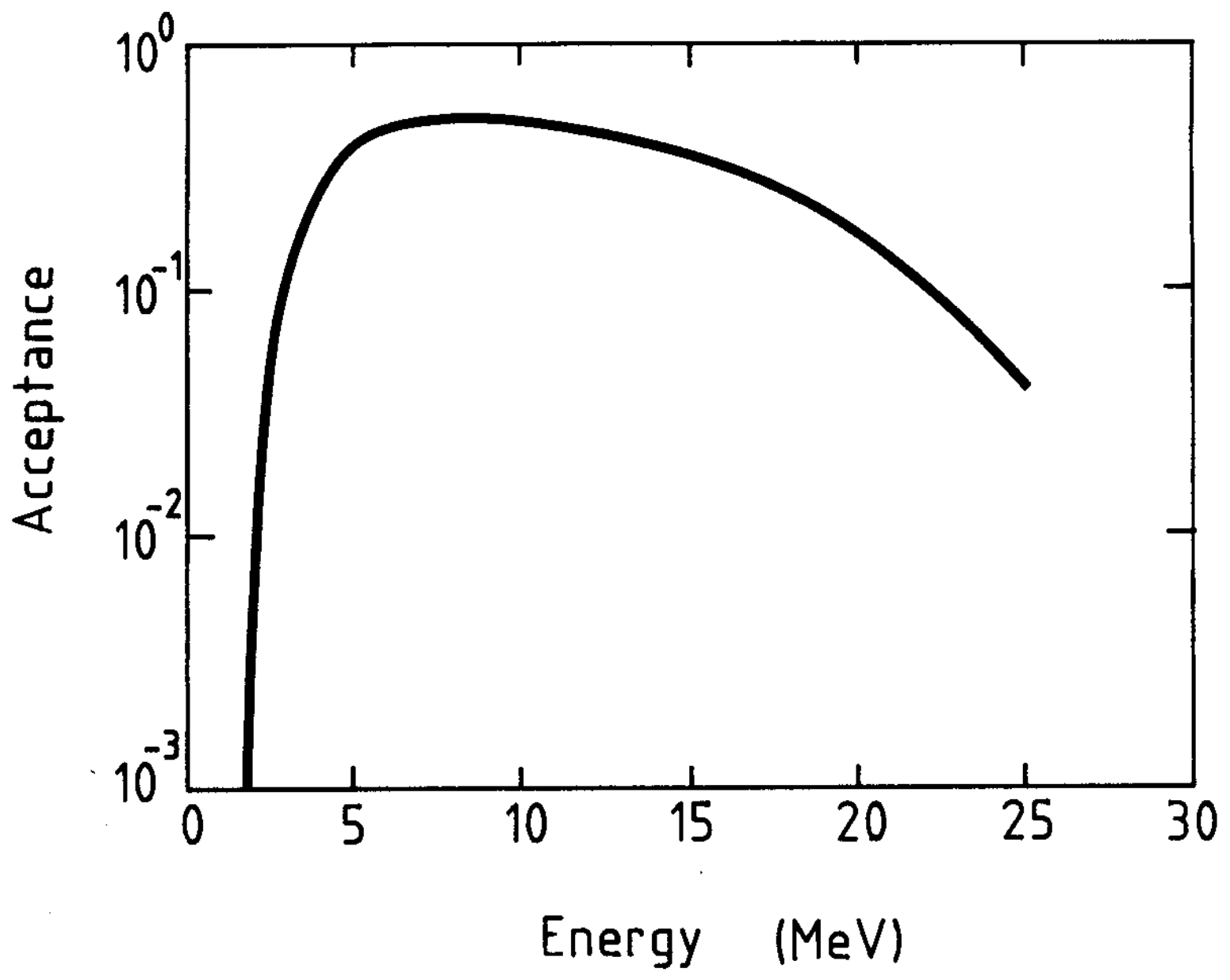

FIG. 14 


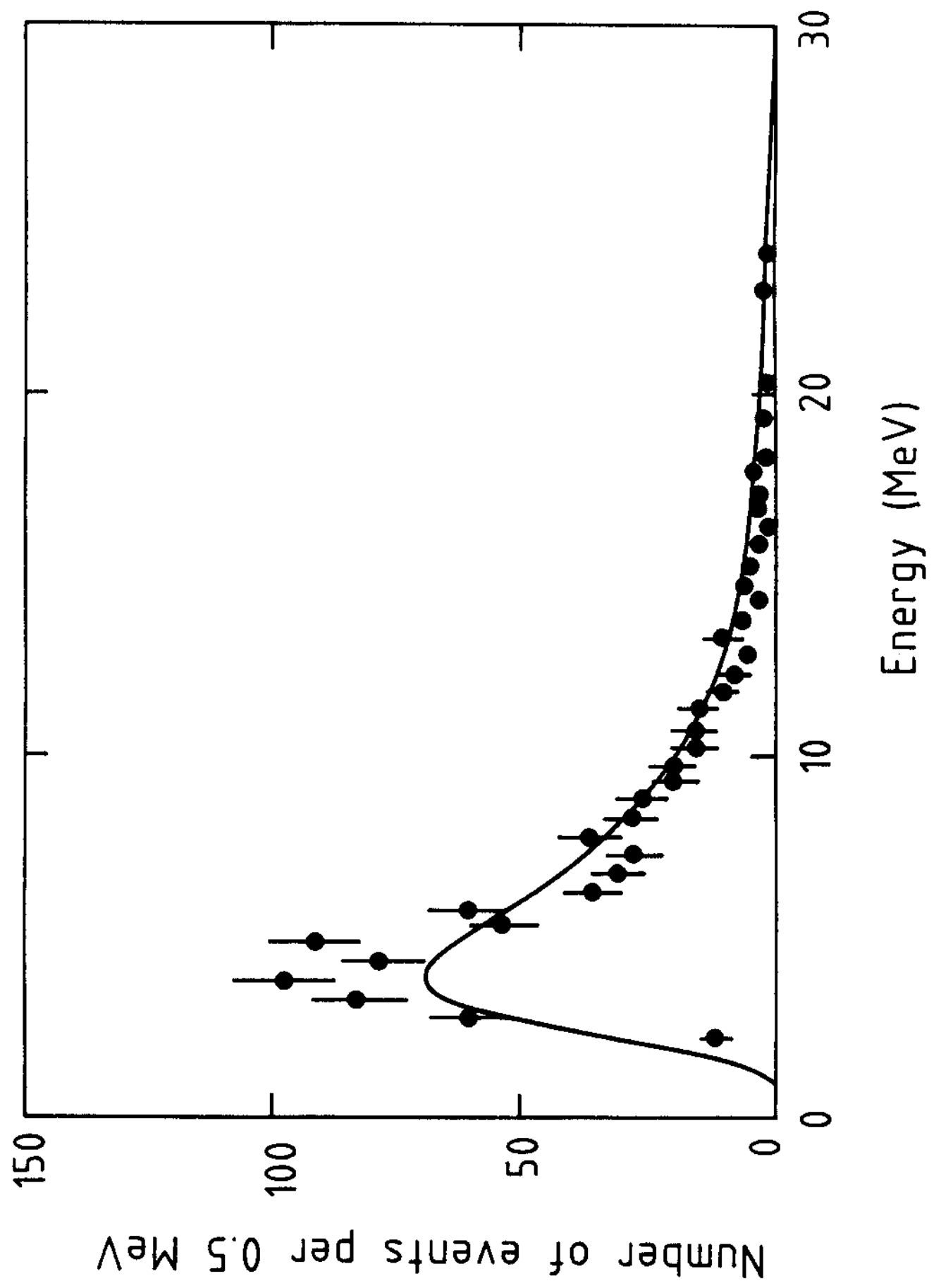

FIG. 15 